\title{
Programas de acceso a la educación superior en tres universidades de la región del Maule. Análisis Documental*
}

\author{
Programs for access to higher education in three universities in the \\ Maule region. Documentary Analysis
}

* Bárbara Letelier Letelier

Letelier, B. (2021). Programas de acceso a la educación superior en tres universidades de la región del Maule. Análisis Documental. Revista Convergencia Educativa, (9), julio, 101-118. DOI: http://doi.org/10.29035/rce.9.101

[Recibido: 24 junio, 2021 / Aceptado: 02 julio, 2021]

\begin{abstract}
RESUMEN
La Educación Superior enfrenta desafíos asociados a calidad y equidad. Una de las propuestas está relacionada con el concepto de inclusión, por lo que el objetivo de la investigación está centrado en el análisis de las directrices de tres programas de acceso a la educación superior de tres universidades chilenas de la región del Maule, por medio de la revisión del estado del arte. Dichos programas son el Programa de Acceso y Acompañamiento Efectivo a la Educación Superior desarrollado en la Universidad de Talca, el Programa de Atracción de Talentos en pedagogía de la Universidad Católica del Maule y el Propedéutico de INACAP. La metodología de trabajo está enmarcada en la revisión de documentos oficiales de los programas de acceso, utilizando investigación cualitativa con un diseño en modalidad de análisis documental. Los resultados arrojan que los programas trabajan con estudiantes de enseñanza media, reforzando competencias transversales y habilidades relacionadas con el proyecto de vida. Para futuras investigaciones, se propone revisar los resultados que han tenido dichos programas en relación con su trabajo, idealmente en algún estudio longitudinal, además de incluir en el análisis las percepciones de los estudiantes en relación con el desarrollo y desempeño de dichos programas.
\end{abstract}

Palabras clave: Inclusión, equidad, calidad, competencias.

\footnotetext{
* El presente trabajo forma parte de una investigación de tesis elaborada durante los años 2019-2020, para el Programa de Magíster en Educación de la Universidad Católica del Maule titulada "Programas de acceso a la educación superior en tres universidades de la región del Maule. Análisis documental. Trabajo dirigido por el Dr. Gerardo Sánchez Sánchez, con la autoría de Bárbara Letelier Letelier.
}

\footnotetext{
* Universidad Tecnológica de Chile, INACAP, sede Talca, Chile. https://orcid.org/0000-0002-3577-6728 bconstanzal@hotmail.com
} 


\begin{abstract}
Higher Education faces challenges associated with quality and equity. One of the proposals is related to the concept of inclusion, so the objective of the research is focused on the analysis of the guidelines of three access programs to higher education of three Chilean universities in the Maule region, through the review of the state of the art. These programs are the Program for Access and Effective Accompaniment to Higher Education developed at the University of Talca, the Program for Attracting Talents in pedagogy at the Catholic University of Maule and the Propedeutic Program at INACAP. The work methodology is framed in the review of official documents of the access programs, using qualitative research with a design in the form of documentary analysis. The results show that the programs work with high school students, reinforcing transversal competences and skills related to the life project. For future research, it is proposed to review the results that these programs have had in relation to their work, ideally in a longitudinal study, in addition to including in the analysis the perceptions of the students in relation to the development and / or performance of said programs.
\end{abstract}

Key words: Inclusion, Equity, Quality, Competences.

\title{
1. INTRODUCCIÓN
}

La educación es un aspecto con el cual se convive desde el nacimiento; la relación de apego con las figuras paternas, las interacciones sociales al interior de la familia y grupos cercanos, el ingreso a la escuela, liceos o universidades, son experiencias que configuran la personalidad y conducta de una persona a través del tiempo (Luengo, 2004).

Bajo ese contexto, se puede decir que la educación es un proceso que acompaña al ser humano a lo largo de su vida, dejando en evidencia que su proyección vital es mucho más clara y provechosa cuando entidades como la escuela se ocupan de fortalecerla (Santana et al., 2010, citado por Santana et al., 2012).

En la actualidad, las entidades de educación superior cumplen el rol de responder a aquellos estudiantes provenientes de contextos vulnerables, que les permite suministrar una oportunidad de integración. En ese contexto, los programas de transición educativa suelen apoyar a los estudiantes que ingresan a la educación superior en escenarios adversos, aun cuando no constituyen la única solución (Leyton et al., 2012).

El aportar al acceso equitativo en la educación superior, se ha transformado entonces en uno de los desafíos que poseen las naciones. La gran mayoría de los sistemas, han tenido deficiencias a la hora de encontrar una forma adecuada, de calidad y con oportunidades masivas, a fin de propiciar la inclusión (Zúñiga et al., 2015).

En definitiva, la inclusión busca generar oportunidades de acceso, permanencia y egreso de la universidad para estudiantes con talento académico, idealmente propuesto para aquellos que son partícipes de contextos vulnerables. Se estima que estos estudiantes puedan ser parte de la educación superior, ya que el talento está repartido de forma igualitaria en la sociedad, por tanto, se deben generar las opciones para fortalecerlo (Lizama et al., 2018). 
De acuerdo con lo expuesto, la presente investigación se enfocó en analizar las directrices de los programas de acceso inclusivo a la educación superior de tres universidades chilenas de la región del Maule, en el contexto de los actuales desafíos de calidad y equidad. Dichos programas son el Programa de Acceso y Acompañamiento Efectivo a la Educación Superior desarrollado en la Universidad de Talca, el Programa de Atracción de Talentos en pedagogía de la Universidad Católica del Maule y el Propedéutico INACAP.

\section{MARCO TEÓRICO}

El concepto de educación puede ser definido como la idea de perfeccionamiento, haciendo alusión a los cambios de estado que poseen las personas, destacando la necesidad de fortalecerse, siendo este un proceso de crecimiento paulatino, donde incluyen factores de índole diversa (del Pozo et al., 2004).

Desde aproximadamente los años 80 hasta la actualidad, se ha puesto cierto énfasis en los procesos de aprendizaje, lo cual llegó paulatinamente a la universalización de la educación escolar y masificación de la educación superior. En torno a lo anterior, se desglosa la importancia social que tiene el aprendizaje, así como también la movilidad social que lo incentiva (Sevilla \& Montero, 2018).

\subsection{Sistema educativo}

El sistema educativo en Chile posee cuatro niveles dentro de su organización: educación parvularia, básica, media y superior. La educación básica y media posee tres modalidades, siendo éstas la educación regular, especial y para adultos, además dentro de la educación media se pueden encontrar tres formaciones diferentes: humanista-científico, técnico profesional y artística.

El sistema educacional es regulado por la Constitución Política de la República de Chile de 1980, la Ley General de Educación (LGE) y la Ley Orgánica Constitucional de Enseñanza (LOCE). Estas dos normativas definen los principios y fines de la educación parvularia, escolar y superior; los derechos y deberes de los integrantes del sistema escolar (incluyendo el rol del Estado); los niveles y modalidades educativas existentes en el sistema; los requisitos para el reconocimiento oficial de los establecimientos que imparten educación parvularia, escolar o superior; y el marco institucional que permite velar por un sistema educativo equitativo y de calidad (MINEDUC, 2017, p. 23).

Respecto de la educación superior, en este nivel, existen tres tipos de proveedores organizados en diferentes instituciones de educación superior según el tipo de certificación que entregan (Ley $\mathrm{N}^{\circ} 20.370$ ), estos son: Universidades, Institutos Profesionales (IP) y Centros de Formación Técnica (CFT).

Las universidades, son instituciones de educación superior que otorgan diversos grados académicos, títulos profesionales con o sin licenciaturas y títulos técnicos. Además, son las únicas que otorgan los grados académicos antes mencionados y se clasifican en privadas y tradicionales. 
Los Institutos profesionales (IP) entregan títulos profesionales y técnicos, sin licenciaturas. Por otra parte, los Centros de formación técnica (CFT) ofrecen títulos técnicos.

Cada una de las instituciones de educación superior, puede someterse a los procesos de acreditación, el cual se ocupa de evidenciar la calidad que posee cada organismo en relación a los procesos educativos. Dicho proceso se realiza por un ente previamente aprobado por el estado (Barrientos \& Araya, 2018).

\subsection{Masificación de la educación superior}

En la década del 80, la tasa de la educación terciaria mostró un aumento progresivo, por tanto, la educación básica y media también había alcanzado altos niveles de cobertura. Lo anterior, conlleva a un nuevo rol protagónico de la educación superior y con ello eleva los niveles de pobreza, traducidos en más años de escolarización (Rama, 2009).

En base a una sociedad globalizada, se ha puesto énfasis en la implementación de un diagrama de aprendizaje constante, lo cual ha ido mutando en la universalización de la educación escolar y masificación de la educación superior, esto a nivel mundial. En torno a lo anterior, se hace énfasis en dos aspectos relevantes, el primero tiene que ver con el énfasis en las funciones sociales que posee el aprendizaje, que se relacionan con el aporte social, laboral, democrático y que llevan a la movilidad social de las personas. Por otra parte, se observa que la función del aprendizaje se relaciona con la economía y productividad de un país o región y como ello debe ir adaptándose a través del tiempo (Sevilla \& Montero, 2018).

En América Latina, se observa que las instituciones de educación terciaria deben desarrollarse y fortalecerse en relación a la pluralidad y plasticidad, para poder asegurar el ingreso y proceso en condiciones de calidad y equidad educativa, esencialmente para sectores vulnerables (Ramos \& López, 2019).

El crecimiento de la educación terciaria se ha centrado principalmente en las universidades y no necesariamente en los institutos profesionales o en los centros de formación técnica, manifiestamente desestimados por parte de los regímenes educativos. Las universidades ofrecen carreras de mayor duración y con costos más elevados en comparación con los IP y CFT; éstas reciben más recursos estatales y beneficios, al igual que mayor atención y revisión de sus procesos, lo que redunda en mayores ingresos a sus egresados (Miranda, 2016).

"La significación de la masificación de la educación superior se reafirma y completa con el reconocimiento del vínculo entre equidad y calidad en este nivel como elementos indivisibles" (Ramos \& López, 2019, p. 294).

Actualmente, alrededor del 50\% de la matrícula de la formación superior técnico profesional está contemplada por Institutos Técnicos Profesionales (IP) y Centros de Formación Técnica (CFT), donde el 
60\% de los estudiantes pertenece al sector más pobre de la población chilena (CINDA, 2008, citado en Barrientos \& Navío, 2015).

La masificación y expansión de la educación superior traen como consecuencia la creación y desarrollo de ciertas instancias e instituciones que consiguen que familias de bajos recursos económicos sean parte de una educación de bajo estándar, observándose que el alto estándar es reservado para la élite (Cepeda et al., 2019).

Según Cepeda et al. (2019) como consecuencia de la masificación de la educación superior, hay instituciones que quedan relegadas a las segundas categorías ofreciendo educación regular; dichas instituciones corresponden principalmente a las entidades que generan una mayor cantidad de vacantes con el único objetivo de asegurar ingresos, pero a su vez, no son reconocidas por la calidad de sus currículos. Este mismo autor refiere que, en el caso de los técnicos y profesionales de nivel medio, la situación varía. Las familias que disponen de mayor capital económico y educativo, acceden a las universidades del Consejo de Rectores de Chile, privilegiando la excelencia en investigación; en el caso en que los padres son trabajadores no calificados o con escolaridad disminuida, hay un mayor ingreso a las universidades no selectivas.

Además, dentro del contexto de educación terciaria, no solo se observan barreras académicas y/o económicas, sino que también psicológicas, asociadas primordialmente con la motivación y contexto cultural, que traen consigo mayor deserción y, por ende, un porcentaje más reducido de titulaciones para estudiantes que vienen de sectores de ingresos bajos (Cepeda et al., 2019).

Según la Organización para la Cooperación y el Desarrollo Económicos (OCDE, 2017) las principales causas de deserción son la falta de vocación, factores económicos y bajo rendimiento académico. Dichos factores tienen que ver con las dificultades de muchas instituciones de educación superior, para adaptarse a la diversa población estudiantil actual.

En ese sentido, "la masificación de la educación superior y con ello el ingreso a la educación terciaria, no significa que los estudiantes aseguran su egreso de dichas instituciones" (Jiménez \& Lagos, 2011, p.133). 


\subsection{Proyecto de vida y juventud}

Otro concepto visible en el contexto de educación terciaria es el proyecto de vida, el cual considera el concepto de vida no solamente como algo biológico, sino que se habla de la habilidad de los seres humanos para trascender en cuanto a su actuar (Daset, 2013).

Dentro del desarrollo del ser humano, existen factores que pueden ser considerados a la hora de revisar su historia vital, uno de ellos es el proyecto de vida, que puede ser definido como:

Una construcción intencional y activa desarrollada a lo largo de la vida; un proceso no lineal concretado en un plan de acción abierto a las oportunidades ofrecidas por el contexto; un proceso complejo donde la inteligencia emocional juega un papel relevante (Romero, 2009, citado en Santana et al., 2012, p. 27).

El proyecto de vida se define como un continuo que ordena y organiza lo que se quiere lograr y obtener personal y socialmente. Dicha construcción, tiene estrecha relación con el entorno social en el cual se fecunda. Por lo tanto, las expectativas y visiones futuras se relacionan con el contexto social e histórico que poseen las personas (García, 2017).

A la hora de elegir o seleccionar una carrera universitaria, los jóvenes poseen varios factores influyentes, entre los cuales se encuentran el contexto de desarrollo y sus características personales, así como su historia de vida y experiencias pasadas (Carrasco et al., 2014).

Entonces, los estudiantes seleccionaran sus carreras observando factores económicos, calidad y prestigio de la institución y progresión académica (García \& Moreno, 2012).

\subsection{Desafíos de las Instituciones de Educación Superior}

Los desafíos orientados a mejorar la educación superior van de la mano con la implementación de medidas que deben llevar a cabo las entidades educativas en el ámbito terciario.

Las reformas y formas de proceder de la educación superior deben mantener relación con contextos que permitan mayor equidad (Ramos \& López, 2019).

Las universidades, tienen como reto ayudar al alumnado más desfavorecido durante sus procesos de admisión y continuidad de estudios, así como también ajustar procedimientos de postulación a la universidad (OCDE, 2018). 


\section{METODOLOGÍA}

La investigación se enmarcó en un enfoque cualitativo (Pérez, 2004), en una revisión documental de diferentes programas de acceso a la educación superior de tres universidades chilenas de la región del Maule. El diseño de investigación asume la modalidad de análisis documental, utilizando materiales oficiales de los programas de acceso inclusivo a la educación superior de tres instituciones que funcionan en la Región del Maule. En caso del programa PACE Utalca, se hizo una revisión de las bases oficiales del programa. En relación a PAT UCM, se hizo una revisión del documento de admisión y requisitos del programa. Y, por último, en el caso del propedéutico Inacap, se hizo una revisión del documento de presentación del programa y material instruccional. Las fuentes mencionadas corresponden a documentos publicados en el sitio web de cada institución educativa, tratándose de documentos públicos, siendo éste el criterio de selección de dichas fuentes.

El análisis de los documentos mencionados se realiza por medio de una lectura y revisión general y luego exhaustiva de éstos. A raíz de la revisión, se confecciona una tabla comparativa con los conceptos claves, creando categorías con los componentes característicos, con el fin de representar sustancialmente lo contenido en los escritos.

Es relevante precisar que dentro de las limitaciones presentadas en esta revisión, es pertinente referir que una de las dificultades hace alusión a la escasa oportunidad de acceso a otro tipo de documentos de la gestión realizada por los programas, ya que, si bien se logró obtener información general en torno a sus finalidades, describir competencias y hacer comparaciones, hubiera sido importante revisar cuáles son las estrategias o técnicas de trabajo que posee cada uno de ellos para cumplir los objetivos que se proponen. Dicha información no se encuentra disponible para utilización general, por tanto, no fue posible verificar estos datos. 


\section{RESULTADOS}

\section{Tabla 1}

Comparación programas de acceso a la educación superior.

$\begin{array}{llll}\text { Categoría } & \text { PACE Utalca } & \text { Propedéutico Inacap } & \text { PAT UCM } \\ & \begin{array}{l}\text { Favorecer el desarrollo integral a través del } \\ \text { reforzamiento de competencias, habilidades }\end{array} & & \\ & \text { transversales (cognitivas, intrapersonales e } & \text { Colaborar en el } & \\ \text { interpersonales) que les ayuden a los } & \text { mejoramiento de los } & \\ \text { Objetivos } & \text { estudiantes en la construcción de sus proyectos } & \text { resultados de aprendizaje } & \text { Posibilitar el acceso a la } \\ & \text { de vida, en su dimensión individual como social. } & \text { de sus alumnos, así como } & \text { universidad, de aquellos } \\ & \text { La idea es que puedan explorar sus vocaciones; } & \text { la orientación académica } & \text { estudiantes destacados } \\ & \text { que aprendan a resolver problemas; que } & \text { y vocacional, para } & \text { que se interesan por la } \\ \text { visualicen que el egreso del liceo representa la } & \text { propender en ellos una } & \text { carrera de pedagogía. }\end{array}$
apertura de diversas posibilidades de trayectoria consecución de Estudios que podrán elegir, para lo cual la preparación Superiores exitosa. recibida les entrega herramientas que necesitarán aplicar.

\begin{tabular}{|c|c|c|c|}
\hline $\begin{array}{l}\text { Población de } \\
\text { trabajo }\end{array}$ & Jóvenes de tercero y cuarto medio. & Jóvenes de cuarto medio. & $\begin{array}{l}\text { Estudiantes de tercero y } \\
\text { cuarto medio, cuyo } \\
\text { promedio está dentro del } \\
10 \% \text { del mejor } \\
\text { rendimiento académico } \\
\text { de su generación y que } \\
\text { tienen orientación o } \\
\text { interés por seguir la } \\
\text { carrera de pedagogía. }\end{array}$ \\
\hline $\begin{array}{l}\text { ¿Qué se } \\
\text { trabaja? }\end{array}$ & $\begin{array}{l}\text { Habilidades transversales (cognitivas, } \\
\text { intrapersonales e interpersonales). }\end{array}$ & $\begin{array}{l}\text { En herramientas para } \\
\text { poder enfrentar la } \\
\text { educación superior } \\
\text { (lenguaje, matemáticas y } \\
\text { proyecto de vida). }\end{array}$ & $\begin{array}{l}\text { Desarrollo personal y } \\
\text { vocación pedagógica, } \\
\text { habilidades } \\
\text { comunicativas y de } \\
\text { pensamiento lógico- } \\
\text { matemático. }\end{array}$ \\
\hline $\begin{array}{l}\text { Lugar de } \\
\text { trabajo }\end{array}$ & Establecimientos educativos seleccionados. & $\begin{array}{l}\text { Se desarrolla en la sede } \\
\text { de Inacap los sábados y } \\
\text { tiene una duración de } 16 \\
\text { sesiones. }\end{array}$ & $\begin{array}{l}\text { Universidad Católica del } \\
\text { Maule, sede San Miguel } \\
\text { en Talca. }\end{array}$ \\
\hline
\end{tabular}

Competencias/habilidades transversales cognitivas, intrapersonales e interpersonales, que se hagan a través de acciones para las cuales Competencias no se prescriben las asignaturas, ni los espacios que fortalece de aprendizaje, ya que se busca que estas competencias/habilidades puedan permear todo el currículo (todas las asignaturas) y todos los espacios formativos.
Responsabilidad, autonomía y trabajo en equipo.
Habilidades pedagógicas académicas y psicoafectivas.
Establecimientos educacionales públicos de enseñanza media joven, diurnos, con altos

Requisitos porcentajes de vulnerabilidad escolar, lo que implica un trabajo con contextos institucionales y socioculturales muchas veces complejos.
Tener un rendimiento académico destacado, dentro del $10 \%$ mejor de su generación y tener interés por la carrera de pedagogía. 
Letelier, B. (2021). Programas de acceso a la educación superior en tres universidades de la región del Maule. Análisis Documental. Revista Convergencia Educativa, (9), julio, 101-118. DOI: http://doi.org/10.29035/rce.9.101

La Tabla 1, muestra las principales características de los programas de acceso a la educación superior, contemplando aspectos esenciales para su conocimiento y comprensión.

La población objetivo prevista por los programas de acceso a la educación, corresponde principalmente a estudiantes de los últimos años de educación secundaria, tercero y cuarto medio en el caso del programa PACE Utalca y PAT UCM; en el de Propedéutico Inacap solamente cuarto medio. Además, en el caso de PAT UCM, los estudiantes deben tener un buen rendimiento académico (estar dentro del 10\% de mejor rendimiento) y tener interés por seguir estudios superiores en pedagogía. En el caso del programa PACE Utalca, si bien todos los estudiantes de tercero y cuarto año pueden participar, no todos tienen la posibilidad de ser un estudiante PACE habilitado, ya que para ello también se considera su puntaje ranking. Lo anterior, hace alusión a lo mencionado por Vergara \& Peredo (2017) quienes dan cuenta de la importancia que tiene el rendimiento académico como un factor relevante ante los resultados académicos futuros, independiente de los puntajes obtenidos en la prueba de acceso a la educación superior.

En relación a las competencias que se pretende fortalecer en los programas, se observa que PACE Utalca se centra principalmente en fortalecer habilidades transversales, las cuáles se dividen en cognitivas, interpersonales e intrapersonales. En torno al Propedéutico INACAP, se fortalecen competencias necesarias para enfrentar la vida universitaria, específicamente vinculadas a las áreas disciplinarias de lenguaje, matemáticas y de una mirada más integral a la proyección de vida. Por último, el PAT UCM, se centra en el trabajo de fortalecimiento de competencias vinculadas al desarrollo personal y vocación de profesor; así como también, habilidades de comunicación y pensamiento lógico matemático. En relación con lo anterior, es importante resaltar que los tres programas focalizan especial interés en fortalecimiento de habilidades necesarias para la inserción de los estudiantes en la vida universitaria, impulsando la progresión académica, sobre todo, hacia estudiantes que poseen condiciones desfavorables previas, con la intención que la nivelación en estas áreas favorezca su ingreso al contexto de la educación terciaria. Ahora bien, es importante considerar que la deserción no solamente se produce por el cambio en el interés inicial, sino que existen factores contextuales, conductuales y motivacionales que influyen, por tanto el dar a conocer a los estudiantes otro contexto educativo -diferente al que ellos han vivido o vivenciado- y mostrar los resultados que ello implica, los orienta a cambiar su conducta futura y obtener motivaciones internas y externas diferentes a las que han formado dado su contexto social (Daset, 2013).

Una visión comparativa de los tres programas de acceso a la educación superior, muestra en términos de similitudes que todos se constituyen como iniciativas o dispositivos de acción que pretenden superar el ámbito de lo cognitivo. Si bien es cierto, en todos ellos, existe un esfuerzo por asegurar conductas de entrada en los ámbito de lenguaje y matemática, también hay un esfuerzo explícito por acciones que buscan fortalecer habilidades para la vida y la proyección de los estudiantes, situación se relaciona con el desarrollo de un proyecto de vida, lo que según Barboza et al. (2017) se corresponde con la familia y la comunicación 
que se puede lograr con ésta; por tanto, allí radica la importancia de considerar éstas habilidades en el trabajo de los programas, ya que al ser estudiantes de contextos vulnerables, propenden a mantener relaciones de comunicación familiar más disminuidas, teniendo menor probabilidad de generar un proyecto de vida claro.

Además, los tres programas se desarrollan en la educación media, tratando de aportar en la etapa previa del ingreso a la educación superior, apostando a la equidad educativa por medio del fortalecimiento de variadas competencias, disminuyendo la brecha académica. Lo anterior, da cuenta de la dificultad existente en la articulación de la enseñanza secundaria hacia la educación terciaria, donde sin duda los estudiantes de establecimientos más vulnerables se ven desfavorecidos; En la misma senda, la educación técnico profesional es la que se ve más perjudicada, ya que en general, no hay una conexión entre sus carreras técnicas de nivel medio con el nivel superior. En consecuencia, el trabajo generado por los programas muestra cómo se desarrolla la educación terciaria, sus exigencias, formas de trabajo e implicancias en general, abriendo así otro camino en la proyección de vida de los estudiantes secundarios lo que puede generar movilidad social en estos y a la larga mayor justicia social (Román, 2013).

De igual forma, dos de los tres programas estudiados (PACE- PAT), entregan un ingreso por vía alternativa a la institución de educación superior, lo que por una parte alude a la idea de que la PSU es una prueba que segrega socioeconómicamente y no permite que estudiantes vulnerables puedan desarrollar su talento de forma igualitaria (González et al., 2017). Desde ese punto de vista, estos dos programas se presentan como claros dispositivos que resaltan mecanismos de justicia social, sustentando la idea de que los talentos están distribuidos de forma igualitaria, independiente de la condición socioeconómica que los estudiantes tengan, lo cual concuerda con la idea base de la inclusión, que permite generar oportunidades de acceso, permanencia y egreso de la universidad, para que los estudiantes tengan la misma oportunidad de desarrollo de competencias (Lizama et al., 2018).

Observando las diferencias, éstas radican principalmente en el rendimiento académico, ya que no es una opción que se considere en el propedéutico. Sumado a ello, el lugar de trabajo también difiere, ya que en el caso de PACE Utalca, se realizan actividades tanto dentro como fuera de la institución de educación superior, lo que no sucede en PAT ni en Propedéutico, ya que los talleres se realizan en los campus correspondientes a cada entidad universitaria. Además, una gran diferencia, es que el programa PAT, prepara a los estudiantes para poder desempeñarse a futuro en un área en particular, que es la pedagogía, y su objetivo es que idealmente los estudiantes ingresen a la universidad; en cambio en los otros dos programas, la preparación es más genérica, no centrándose en una carrera en particular y teniendo como principales objetivos el favorecer el desarrollo integral (PACE) y mejoramiento de aprendizajes, orientación académica y vocacional; de lo anterior se deduce, que de cierta forma para acceder al PAT ya debe existir cierta claridad 
de la proyección de vida en torno al desarrollo de un área profesional, no así en PACE Utalca y Propedéutico INACAP.

Dentro de las principales directrices de estos programas, se encuentra la etapa formativa de los estudiantes y la ampliación de equidad y acceso a la educación terciaria. Respecto a la etapa formativa, es que se orientan al trabajo con estudiantes de educación media, dando lugar al fortalecimiento de habilidades y competencias transversales para potenciar los recursos de los estudiantes, pensando en su posible ingreso a la educación superior, a fin entregar confianza a sus talentos y esfuerzos y disminuir la brecha académica y social existente.

Dicha preparación, responde a lo propuesto por Sevilla \& Montero (2018), quienes plantean que el desarrollo de habilidades transversales y el aprender a aprender, son primordiales para la educación, o como lo mencionan Montes \& Machado (2014), los que concuerdan que los estudiantes de educación terciaria deben ser preparados para desarrollarse en el contexto social en el cual participan, por tanto, requieren habilidades diversas para lograrlo.

Esto también, hace alusión a la proyección vital de los jóvenes, la que según Carrasco et al. (2014) se fortalece y se orienta a medida que se muestra otro contexto a los estudiantes y se les motiva a reconocer sus propias aptitudes, abordando así algunos de los factores que influyen en la construcción este proyecto vital, por consiguiente, en la alternativa de seguir una carrera universitaria y/o de la selección de ésta.

En relación a la ampliación de equidad y acceso a la educación terciaria, se refiere a que los programas se enfocan en conjugar la integración social y académica, ya que aquellos estudiantes de contextos vulnerables se ven afectados en las posibilidades de expandir su interacción social (Leyton et al., 2012).

Esta posibilidad de expandir su interacción social propende a desarrollar a largo plazo una equidad educativa y se presenta entonces como una condición sumamente necesaria para mejorar la movilidad social, sobre todo en aquellos grupos de población menos privilegiada, donde la educación se transforma en un arma importante para mejorar las condiciones de vida (OCDE, 2017).

Sintetizando, el desarrollo y gestión que pueden desempeñar los programas va orientado a generar otras instancias de comunicación y motivación en los estudiantes, lo cual tiende a un desarrollo más claro de sus proyectos de vida y por consiguiente mayores probabilidades de movilidad social (Leyton et al., 2012). Además, estos programas generan actualmente un aporte a disminuir esa brecha, utilizando una vía de acceso diferente a la PSU, que se presenta como un instrumento de evaluación que segrega socioeconómicamente.

Bajo ese prisma, las instituciones de educación superior, deben generar instancias responsables para facilitar la progresión académica y dar tiempos de adecuación, sobre todo a estudiantes de contextos vulnerables ya que debe entenderse la equidad e inclusión como derechos, definiendo que el proceso 
académico de un estudiantes es responsabilidad personal, como de la institución de la cuál es parte, teniendo ésta la obligación de generar condiciones necesarias para la obtención de aprendizajes (Sobrero, 2018).

Sumado a lo anterior, es relevante que las universidades creen políticas de inclusión que tengan un impacto masivo ante la comunidad, ya que, si esto no ocurre, es un trabajo puramente interno y poco provechoso para el bienestar social general (Lizama et al., 2018). En definitiva, las reformas y formas de proceder de la educación superior deben ir de la mano con contextos que permitan mayor equidad en dicho nivel educativo (Ramos \& López, 2019).

Además, las instituciones deben incluir en su proyección futura cambios y actualizaciones en pro del aprendizaje, ya que este impactará directamente en desempeño profesional y la estructura del empleo, la que, con el paso del tiempo, exigirá que la educación terciaria se expanda y haga cambios en relación a las competencias requeridas por el mercado laboral (OCDE, 2018).

En cuanto a las habilidades relacionadas con el mercado laboral, también se espera que las instituciones de educación terciaria identifiquen en los estudiantes sus proyecciones y capacidades y potencien su desarrollo para que éstos desempeñen un rol en la comunidad (Van de Werfhorst \& Mijs, 2010, citado por Leyton et al., 2012).

Respecto a las diferencias entre estos programas, estas se relacionan con el lugar donde se ejecutan las actividades (colegio versus instituciones de educación terciaria), criterios de acceso (colegios seleccionados por vulnerabilidad versus postulación) y las habilidades trabajadas (unas transversales y otras orientadas directamente a la carrera de elección del estudiante). Sin embargo, estas discrepancias, los lineamientos de los programas, buscan proveer otro sistema de acceso y potenciar herramientas para la educación en los estudiantes.

A lo largo de la investigación, se ha revisado la temporalidad del sistema educativo chileno respecto a la educación terciaria: procesos de juventud y proyección vital, masificación de la educación superior, así como también los desafíos que debe asumir la educación y las instituciones que la imparten.

En esa perspectiva, la revisión teórica ha puesto de manifiesto la importancia que posee en la educación terciaria la equidad, la inclusión, y la calidad educativa, puesto que en la actualidad constituyen las grandes y urgentes demandas que enfrenta el sistema educativo en su conjunto. Ya que, si bien se están implementando acciones para disminuir la brecha económica (la gratuidad para familias vulnerables, por ejemplo), aún se debe avanzar en reducir las diferencias sociales y académicas, lo cual afecta profundamente en la progresión académica y egreso exitoso de las carreras universitarias por parte de los estudiantes (OCDE, 2017). 
En ese escenario surgen los programas de acceso inclusivo, los cuales tienen en términos generales el propósito de lograr una mayor equidad en la educación superior, enfocándose en el potencial que muestran los estudiantes desde la educación escolar. Cuando se habla de inclusión, se piensa la idea de generación de oportunidades de acceso, permanencia y egreso de la universidad para estudiantes de forma igualitaria.

Los resultados del estudio documental que ha tenido como objetivo general analizar tres programas de acceso inclusivo a la educación superior de tres universidades chilenas de la región del Maule, permite establecer las siguientes conclusiones:

\section{CONCLUSIONES}

En primer lugar, en relación a las finalidades y población objetivo que instauraron los programas de las universidades de estudio, se verificó que los programas analizados se orientan a fortalecer las habilidades transversales en los estudiantes y prepararlos para su desempeño en la etapa universitaria. Además, dos de ellos se centran principalmente en la idea de que los talentos están democráticamente repartidos en la sociedad, indistintamente de la situación económica que los estudiantes posean. Dado lo anterior, los esfuerzos de los programas analizados se centran en la educación media, generando un trabajo con estudiantes de los últimos años de educación secundaria

En segundo lugar, en torno a las principales competencias que se pretende fortalecer por medio de los programas de acceso a la educación superior de tres universidades chilenas de la región del Maule, se evidenció que estos se centran en habilidades para la vida, priorizando no solamente lo cognitivo, sino que también habilidades de carácter transversal, las cuales van acorde a los requerimientos de la sociedad profesional.

En tercer lugar, al generar una comparación entre los programas de acceso a la educación superior de tres universidades chilenas de la región del Maule a fin de reconocer puntos de encuentro y diferencias entre sí, se observó que estos programas tratan de fortalecer las competencias de los estudiantes antes del ingreso a la educación superior, trabajando con estudiantes de educación media, sustentando la justicia social, permitiendo generar oportunidades de acceso, sin que ello signifique un esfuerzo decidido por asegurar su permanencia y egreso de la educación terciaria. Respecto a las diferencias entre estos programas, estas se relacionan con el lugar donde se ejecutan las actividades, criterios de acceso y las habilidades trabajadas. En cuanto a las actividades, las diferencias son que PACE Utalca utiliza las dependencias de la universidad y las instituciones educativas (liceos) para generar las actividades, PAT UCM desarrolla las actividades en sus dependencias, al igual que Propedéutico INACAP. En relación al acceso, las diferencias muestran que hay liceos seleccionados para trabajar dada su vulnerabilidad (PACE Utalca), y en otros el trabajo se genera por postulación (Propedéutico INACAP y PAT UCM). Por último, en cuanto a las habilidades, las diferencias radican en que PACE Utalca y Propedéutico INACAP se orientan más 
al trabajo de habilidades transversales y PAT UCM a habilidades relacionadas directamente con la carrera de pedagogía.

Entonces, luego de la revisión del estado del arte y el análisis de los programas, se puede formular que:

Las políticas nacionales vinculadas a la educación superior deben propender a proporcionar herramientas que permitan no sólo el acceso, sino que también a la permanencia, egreso y titulación oportuna de la educación superior. Además, dar continuidad al trayecto que los estudiantes deben sobrellevar en la institución de educación superior. No es suficiente con el ingreso, ya que la idea fundamental es dar movilidad social a los estudiantes, especialmente de contextos vulnerables, por tanto, es necesario acompañar hasta la titulación.

Más que solamente programas de acceso, dichos dispositivos deberían ser transversales en la educación media, es decir, generarse instancias para el desarrollo de una proyección vital y del fortalecimiento de habilidades transversales como parte del proceso de enseñanza media, especialmente para aquellos estudiantes que no tengan claridad en su área vocacional o de interés laboral a futuro. Lo anterior, daría pie para una construcción más informada de su vida futura, así como también mayor orientación y ayuda para aquellos estudiantes cuyo entorno familiar no disponga de los recursos socioculturales para fomentar un impacto positivo en la construcción de su proyecto de vida.

Como otra medida de apoyo a la inclusión educativa, se hace necesario crear programas de acceso inclusivo orientados a carreras específicas, gestionando el interés vocacional de los estudiantes desde la educación secundaria. Lo anterior da lugar a crear más programas como PAT UCM, que fortalezcan habilidades específicas según los intereses de los estudiantes, lo cual también aportaría en la mantención de estos en su carrera, a un proyecto de vida más claro, a mejores oportunidades de desarrollo según los recursos personales de cada uno, teniendo como resultado profesionales con más vocación e interés por su desempeño en el mundo laboral.

Existe un desafío pendiente, que es el fortalecer las habilidades de aquellos docentes que reciben y trabajan directamente con los estudiantes en el primer año de ingreso, para que éstos generen ambientes de aprendizaje que promuevan la comprensión conductual, emocional, relacional, inclusiva y académica del desafío que implica la transición entre educación secundaria y terciaria, pero por sobre todo facilitando la integración de conocimientos, habilidades y actitudes que caracterizan a un ciudadano activo y participativo.

Todo con la finalidad de potenciar la progresión académica a partir de la claridad de la proyección trazada por los estudiantes y, por tanto, una mayor precisión en su proyección vital y desempeño profesional futuro, lo que disminuiría la tensión constante de los jóvenes en su elección por la educación versus el mundo del trabajo (Sepúlveda, 2013). 
Respecto a las líneas investigativas que se pueden generar luego de este análisis, hay dos lineamientos claros: Por un lado, generar un estudio longitudinal, respecto a los resultados e impacto que han tenido los programas de acceso respecto al ingreso, permanencia y egreso de los estudiantes a la educación terciaria, de preferencia, este estudio considere información que provenga de los estudiantes con los cuales se trabaja, en relación a la pertinencia e interés de su participación en dichas instancias; así como también el impacto, que ellos reconocen, tiene el desarrollo de dichos programas en su progresión académica, perfeccionamiento de competencias y movilidad social.

Y, por otro lado, integrar instancias para fomentar la reflexión y potenciación de habilidades transversales e inteligencia emocional en el mundo actual en la educación media, lo cual trazaría ideas más claras en la proyección de vida de los estudiantes, donde dichos establecimientos educacionales actúen como precursores del acceso a la educación superior y que de esta forma articulen dichas ideas con la educación terciaria y en este caso con los programas de acceso inclusivo de forma paralela.

En síntesis, si bien se está haciendo un gran esfuerzo por parte de las entidades educativas y del gobierno por crear instancias de inclusión y equidad en la educación terciaria, estas no son suficientes para responder a las necesidades que poseen los estudiantes, ya sea por su condición socioeconómica, por su condición sociocultural o por la formación con la que cuentan. Si bien se está avanzando también en relación al financiamiento, no es suficiente con ingresar a estudiantes a la universidad, se requiere acompañar dichos procesos para evitar trayectos poco exitosos. Los estudiantes deben ser preparados en relación a conocimientos y habilidades que serán parte de su desempeño profesional, pero también se debe considerar sus competencias transversales asociadas al contexto social del cual provienen y al cual se van a enfrentar.

En relación a las limitaciones presentadas en esta revisión, se puede referir que una de las dificultades hace alusión a la escasa oportunidad de acceder a otro tipo de documentos de la gestión realizada por los programas, ya que si bien fue posible obtener información general en torno a sus finalidades, describir competencias y hacer comparaciones, hubiera sido importante revisar cuáles son las estrategias o técnicas de trabajo que posee cada uno de ellos para cumplir los objetivos que se proponen. Dicha información no se encuentra disponible para utilización general, por tanto, no fue posible suscribir estos antecedentes. 


\section{REFERENCIAS BIBLIOGRÁFICAS}

Barboza, M., Moori, I., Zárate, S., López, A. Muñoz, K., \& Ramos, S. (2017). Influencia de la dinámica familiar percibida en el proyecto de vida en escolares de una institución educativa de Lima. Psicología Escolar e Educacional, 21(2), 157-166. https://doi.org/10.1590/2175-3539201702121094

Barrientos, C., \& Navío, A. (2015). Formador de la educación superior técnico profesional: Percepciones sobre su trabajo docente. Estudios Pedagógicos, (41), 45-61. http://dx.doi.org/10.4067/S071807052015000100003

Barrientos-Oradini, N., \& Araya-Castillo, L. (2018). Educación Superior en Chile. Revista Aletheia, 10(2), 80 109. http://dx.doi.org/10.11600/21450366.10.2aletheia.80.109

Cepeda, M., Bizama, M., Casanova, D., \& Oliva, C. (2019). Propuesta metodológica para la obtención de un Indicador Nacional de Vulnerabilidad Académica: un insumo para políticas públicas y procesos de acreditación con foco en la equidad. Cuadernos de Investigación en Aseguramiento de la Calidad, (15). https://www.cnachile.cl/SiteAssets/Paginas/cuadernos/Cuaderno\%2015.pdf

Carrasco, E., Zuñiga, C., \& Espinoza, J. (2014). Elección de carrera en estudiantes de nivel socioeconómico bajo de universidades chilenas altamente selectivas. Calidad en la educación (40), 95-128. https://dx.doi.org/10.4067/S0718-45652014000100004

Daset, L. (2013). Proyecto de vida: una propuesta terapéutica que se enmarca en la psicología positiva. ECOS, $\begin{array}{llll}\text { Estudos Contemporâneos } \quad d a \quad \text { Subjetividade, } & 3(2), \quad \text { 276-290. }\end{array}$ http://www.periodicoshumanas.uff.br/ecos/article/view/1253

Del Pozo, M., Álvarez, J., Luego, J., \& Otero, E. (2004). Teorías e instituciones contemporáneas de educación. Biblioteca Nueva.

García, K. (2017). Construcción de Proyectos de Vida Alternativos (PVA) en Urabá, Colombia: papel del sistema educativo en contextos vulnerables. Estudios Pedagógicos, 43(3), 153-173. https://dx.doi.org/10.4067/S0718-07052017000300009

García, J., \& Moreno, C. (2012). Factores seleccionados al considerar una universidad. Revista mexicana de investigación educativa, 17(52), 287-305. https://www.redalyc.org/articulo.oa?id=14023076012

González, P., Arancibia V., \& Boyanova, D. (2017). Talento académico, vulnerabilidad escolar y resultados en la prueba de selección universitaria. Estudios Pedagógicos, 43(1), 171-191. http://dx.doi.org/10.4067/S0718-07052017000100011

Jiménez, M., \& Lagos, F. (2011). Nueva geografía de la educación superior y de los estudiantes, una cartografía del sistema chileno, su actual alumnado y sus principales tendencias. Universidad San Sebastián. https://aequalis.cl/wp-content/uploads/2020/06/nueva-geografia-de-la-educacion-superior-yde-los-estudiantes.pdf 
Leyton, D., Vásquez, A., \& Fuenzalida, V. (2012). La experiencia de estudiantes de contextos vulnerables en diferentes instituciones de educación superior universitaria (IESU): resultados de investigación. Calidad de la educación, (37), 61-97. https://dx.doi.org/10.4067/S0718-45652012000200003

Lizama, O., Gil, F., \& Rahamer, B. (2018). La experiencia de la inclusión en la educación superior en Chile. Universidad de Santiago de Chile. https://paiep.usach.cl/sites/paiep/files/documentos/EXPERIENCIA_DE_LA_INCLUSION_LIVilovepdf-compressed.pdf

Luengo, J. (2004). Teorías e instituciones contemporáneas de educación. Biblioteca Nueva

Ministerio de Educación (2017). Revisión de las Políticas Educativas en Chile. https://centroestudios.mineduc.cl/wp-content/uploads/sites/100/2017/06/CBR_MineducWEB.pdf

Miranda, G. (2016). Paradojas de la Modernización del Sistema Universitario Chileno. Revista Latinoamericana, 15(45), 345-361. https://dx.doi.org/10.4067/S0718-65682016000300017

Montes, N., \& Machado, F. (2014). Formación y desarrollo de competencias en la educación superior cubana. Humanidades Médicas, 14(1), 145-159. http://scielo.sld.cu/pdf/hmc/v14n1/hmc10114.pdf

Organización para la Cooperación y el Desarrollo Económicos (2017). Evaluaciones de Políticas Nacionales de Educación. Educación $\quad$ en $\quad$ Chile. http://archivos.agenciaeducacion.cl/Educacion_en_Chile_OCDE_Nov2017.pdf

Organización para la Cooperación y el Desarrollo Económicos (2018). Revisiones de la OCDE sobre educación: principales conclusiones. https://politicaspublicas.uc.cl/wpcontent//uploads/2018/05/Seminario_Desafios_Educacion_Chile_Paulo_Santiago_30Mayo2018_ FINAL.pdf

Pérez, G. (2004). Investigación cualitativa. Retos e interrogantes. La Muralla.

Rama, C. (2009). La tendencia a la masificación de la cobertura de la educación superior en América Latina. Revista Iberoaméricana, (50), 173-195. https://doi.org/10.35362/rie500668

Ramos, G., \& López, A. (2019). Masificación, equidad, educación superior: la universalización de la educación superior cubana. Ensaio: Avaliação e Políticas Públicas em Educação, 27(103), 291-316. https://doi.org/10.1590/S0104-40362019002701783

Román, C. (2013). Más programas propedéuticos en Chile. El discurso de los estudiantes en la Universidad Católica Silva Henríquez. Estudios Pedagógicos, 39(2), 263-278. http://dx.doi.org/10.4067/S071807052013000200017 
Santana Vega, L., Feliciano García, L., \& Santana Lorenzo, A. (2012). Análisis del proyecto de vida del alumnado de educación secundaria. Revista Española de Orientación y Psicopedagogía, 23(1), 2638. https://www.redalyc.org/pdf/3382/338230790004.pdf

Sevilla, P., \& Montero P. (2018). Articulación de la educación técnica formal, no formal e informal: Garantías de calidad para un continuo educativo. Cuadernos de Investigación en Aseguramiento de la Calidad, (9).

\section{https://www.researchgate.net/publication/328198394 Articulacion de la educacion tecnica fo rmal no-formal e informal garantias de calidad para un continuo educativo}

Sepúlveda, L. (2013). Juventud como transición: elementos conceptuales y perspectivas de la investigación en el tiempo actual. Última década, 21(39), 11-39. http://dx.doi.org/10.4067/S071822362013000200002

Sobrero, V. (2018). Hacia una docencia inclusiva en la Educación Superior: La investigación sobre la propia práctica docente como herramienta de transformación. Revista Chilena de Pediatría, (89), 7-9. https://dx.doi.org/10.4067/S0370-41062018000100007

Vergara-Díaz, G., \& Peredo-López, H. (2017). Relación del desempeño académico de estudiantes de primer año de universidad en Chile y los instrumentos de selección para su ingreso. Revista Educación, 41(2), 95-104. http://dx.doi.org/10.15517/revedu.v41i2.21514

Zúñiga, C., Redondo, J., López, M., \& Santa Cruz, E. (Ed). (2015). Equidad en la educación superior: desafíos y proyecciones en la experiencia comparada. El Desconcierto. https://www.uach.cl/uach/_file/ed_superior-modelos-59df849066a6f.pdf

\section{Datos de correspondencia}

Mg. Bárbara Constanza Letelier Letelier

Universidad Tecnológica de Chile, INACAP, sede Talca.

Talca, Chile.

Dirección postal: 3460000

ORCID ID: https://orcid.org/0000-0002-3577-6728

Email: bconstanzal@hotmail.com

Esta obra está bajo una Licencia de Creative Commons Reconocimiento-NoComercial-

Compartirlgual 4.0 Internacional. 